\title{
Analytical solution of groundwater waves in unconfined aquifers with sloping boundary
}

\author{
SELVA BALAJI MUNUSAMY and ANIRBAN DHAR* \\ Department of Civil Engineering, Indian Institute of Technology Kharagpur, Kharagpur 721302, India \\ e-mail: anirban@civil.iitkgp.ernet.in; anirban.dhar@gmail.com
}

MS received 11 July 2016; revised 7 February 2017; accepted 18 May 2017; published 29 July 2017

\begin{abstract}
A new analytical solution is derived for tide-driven groundwater waves in coastal aquifers using higher-order Boussinesq equation. The homotopy perturbation solution is derived using a virtual perturbation approach without any pre-defined physical parameters. The secular term removal is performed using a combination of parameter expansion and auxiliary term. This approach is unique compared with existing perturbation solutions. The present first-order solution compares well with the previous analytical solutions and a 2D FEFLOW solution for a steep beach slope. This is due to the fact that the higher-order Boussinesq equation captures the streamlines better than ordinary Boussinesq equation based on Dupuit's assumption. The slope of the beach emerges as an implicit physical parameter from the solution process.
\end{abstract}

Keywords. Analytical solutions; coastal aquifers; homotopy perturbation; phreatic aquifers; second-order theory.

\section{Introduction}

Groundwater from the coastal aquifers contributes immensely to meet the freshwater demands of the coastal population. Hence, the understanding of the interaction between the seawater fluctuations due to tides and the groundwater table becomes essential. Analytical solution of tide-aquifer interaction is important from field application [1] point of view. In the present work, the water table wave propagations in coastal aquifers are solved using a homotopy perturbation method with simple harmonic sinusoidal functions representing the tidal fluctuations.

The propagation is a highly nonlinear process with attenuated amplitude and phase lag with increasing distance from the seaside boundary [2]. The fluctuations due to tidal oscillations are negligible at an asymptotic distance from the beach face. Parlange et al [3] used a perturbation approach based on Dagan's [4] shallow-flow theory to derive the Boussinesq equation. Parlange et al [3] used an amplitude parameter to provide the solution for water table fluctuations due to simple harmonic tidal variations in a coastal aquifer. The amplitude parameter is defined as $A / D$, where $A$ is the amplitude of the fluctuation and $D$ is the mean sea level above the impervious

Electronic supplementary material The online version of this article (doi:10.1007/s12046-017-0695-8) contains supplementary material, which is available to authorized users.

*For correspondence aquifer bottom. The solution by Parlange is applicable for vertical beach face. However, in natural systems the beach face is normally sloped. Nielsen [2] used a linearized Boussinesq equation to provide solutions for a coastal aquifer with sloping beach face. Nielsen [2] assumed a fixed location boundary condition and the perturbation parameter included the slope of the beach face. Li et al [5] introduced a coordinate transformation for moving-boundary effects due to tidal water level variations along the beach slope. Teo et al [6] and Jeng et al [7] derived solutions for water table waves due to moving boundary effects in a sloping beach face using non-dimensional perturbation parameters. However, these solutions are restricted to low orders due to secular terms generation in the solution process. Secular terms make the solutions unbounded. Song et al [8] and Kong et al [9] removed the secular terms from the solution using perturbation expansions for the boundary condition. However, the perturbation expansion introduces small corrections to the seaside boundary. Cartwright et al [10] presented an analytical solution considering dual length scales into account. This approach of using imaginary wave numbers is non-physical as they are determined based on least squares fitting. Stojsavljevic et al [11] derived a higher-order, semi-analytical solution for vertical beach face using two non-dimensional perturbation parameters by proposing an ansatz. Fenton [12], Nielsen et al [13], Liu and Wen [14] and Castro-Orgaz and Dey [15] derived a second-order Boussinesq equation with different approaches. Fenton [12] derived the second-order Boussinesq equation based on shallow-flow theory, while Nielsen et al [13] derived the same equation assuming linearly varying vertical velocity 
between the free surface and aquifer bottom. The correction for the deviation from the hydrostatic pressure due to vertical velocity in the pressure distribution is used to derive the second-order Boussinesq equation for intermediate aquifer depths. Liu and Wen [14] derived the second-order equation by a perturbation technique based on shallowness parameter and nonlinearity parameter, which represented the surface displacement. Castro-Orgaz and Dey [15] used Picard's iteration technique to derive a second-order Boussinesq equation based on Cauchy-Reimann conditions.

Homotopy analysis method (HAM) for groundwater dynamics was first used by Song and Longbin [16]. In the present work, the second-order Boussinesq equation is used to derive a new analytical solution for water table waves due to tidal boundary condition. The solution is derived for a coastal aquifer with sloping beach face as the interface between seawater and aquifer, by incorporating moving-boundary effects. The homotopy perturbation method is used to obtain a dimensional solution with virtual parameters. It has an advantage over conventional perturbation approach in terms of perturbation parameter specification. The secular terms generated in the solution are eliminated using parameter expansion and an auxiliary term.

\section{Methodology}

The second-order Boussinesq equation for groundwater flow in a homogeneous and isotropic unconfined aquifer can be given as [13] (figure 1) follows:

$$
h,_{t}=K / \eta_{e}\left(h h,_{x}+h^{2} h,_{x} h,_{x x}+\frac{1}{3} h^{3} h,{ }_{x x x}\right),{ }_{x}
$$

where $h$ is the local water table height, $K$ is the hydraulic conductivity of the aquifer, $\eta_{e}$ is the effective porosity of the aquifer, $h,{ }_{t}$ is the partial differential of water table height $h$ with respect to $t, h,{ }_{x}$ is the partial differential of $h$ with respect to $x,(),{ }_{x}$ is the partial differential of the expression inside the braces with respect to $x, x$ is the longitudinal coordinate with $x=0$ at the interface of mean

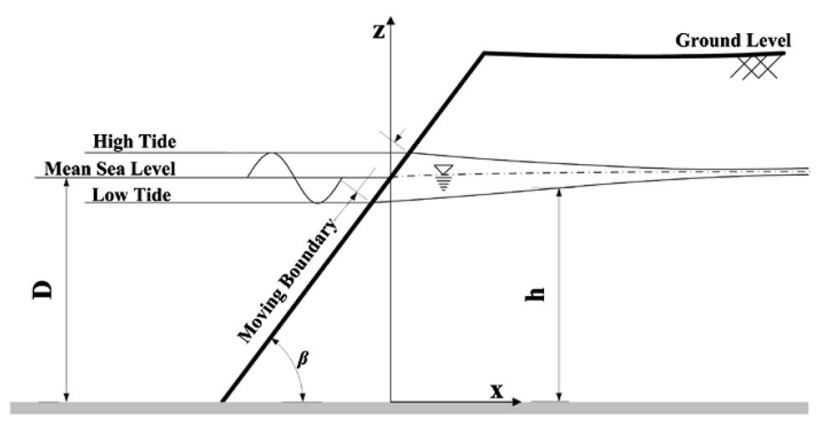

Figure 1. Vertical cross-section of a typical coastal unconfined aquifer for the tidal influence on groundwater table. sea level and aquifer and $t$ is the time. The regional freshwater flux, recharge due to rainfall, seepage face and capillary effects are assumed to be negligible for deriving the simplified analytical solution. The partial derivatives with respect to $x$ in the right-hand side of Eq. (1) are nonlinear. The tidal boundary condition is represented as a cosine function with $A$ and $\omega$ as the amplitude and the angular frequency of the cosine wave, respectively. In reality, seepage face formation is common for tide-aquifer interaction problems. To simplify the complexity of the problem, seepage face formation is neglected [2-11]. The tidal boundary condition can be written as a cosine function as follows:

$$
h(0, t)=D+A \cos [\omega t]
$$

where $D$ is the mean sea level above aquifer bottom. The fluctuations are negligible at a distance far inland from the seaside boundary. Hence, the inland boundary condition can be written as

$$
h,\left.x\right|_{x \rightarrow \infty}=0 .
$$

The intersection between seawater and aquifer moves along the slope of the beach face due to tidal variation. The coordinate transformation introduced by [6] can be used to fix the moving boundary as follows:

$$
\begin{gathered}
x_{0}(t)=\cot (\beta) A \cos (\omega t), \\
x^{*}=x-x_{0}(t) .
\end{gathered}
$$

On substituting Eq. (5) in Eq. (1), the governing equation becomes

$$
\begin{aligned}
h_{, t}= & K / \eta_{e}\left(h h_{x^{*}}+h^{2} h,_{x^{*}} h_{x_{x^{*}} x^{*}}+\frac{1}{3} h^{3} h_{x^{*} x^{*} x^{*}}\right)_{, x^{*}} \\
& -A \omega \cot [\beta] \sin [\omega t] h_{x^{*}} .
\end{aligned}
$$

The last term in the RHS of the equation represents slope effects.

\subsection{The homotopy perturbation method}

He [17] developed the homotopy perturbation method by combining the homotopy technique with traditional perturbation method to solve nonlinear ordinary/partial differential equations (PDEs). The homotopy equation is constructed with virtual parameters in a way such that the equation deforms from the original nonlinear equation to a linear approximate equation when the virtual parameters change from one to zero. The homotopy equation is solved assuming an approximate solution in perturbation form using the virtual parameters similar to traditional perturbation techniques. The homotopy equation for the groundwater waves in aquifers (Eq. (1)) can be constructed by embedding small parameters $p_{1}$ and $p_{2}$ as follows: 


$$
\begin{gathered}
h_{, t}=\underbrace{0 . h_{x^{*} x^{*}}}_{\text {virtual term }}+p_{1} K / \eta_{e}\left(h h_{x^{*}}+h^{2} h_{, x^{*}} h_{x^{*} x^{*}}\right. \\
\left.+\frac{1}{3} h^{3} h_{x^{*} x^{*} x^{*}}\right),_{x^{*}} \\
+p_{2}\left(-s_{p} \sin [\omega t] h_{x^{*}}\right)+\underbrace{\alpha p_{1}\left(1-p_{1}\right) h_{x^{*}}}_{\text {auxiliary term }} \\
s_{p}=A \omega \cot [\beta]
\end{gathered}
$$

where $p_{1}$ and $p_{2}$ are the virtual perturbation parameters and $\alpha$ is the coefficient of the auxiliary term. The equation deforms from the original nonlinear equation to an approximate linear equation when the embedding parameters change from one to zero $\left(p_{1} \rightarrow 0, p_{2} \rightarrow 0\right)$ as per the homotopy definition. The parameter $p_{1}$ is used for nonlinear terms from the second-order Boussinesq equation and $p_{2}$ is used for the term representing slope effects. The virtual term and auxiliary term [18] are added to eliminate the secular terms. The auxiliary term becomes zero when the virtual parameter $p_{1}$ is equal to

$$
0=w_{0,0}-\sum_{m=0}^{N_{p}} \sum_{n=0}^{N_{p}} p_{1}^{m} p_{2}^{n} w_{m, n} B_{\left[m+n \leq N_{p}\right]} B_{[m+n>0]} .
$$

The secular terms generated by second and third nonlinear terms can be eliminated using the auxiliary term in the homotopy equation. The coefficient of the auxiliary term $\alpha$ can also be expanded in the following form:

$$
\alpha=\alpha_{0,0}+\sum_{m=0}^{N_{p}} \sum_{n=0}^{N_{p}} p_{1}^{m} p_{2}^{n} \alpha_{m, n} B_{\left[m+n \leq N_{p}\right]} B_{[m+n>0]} .
$$

Substituting Eqs. (9)-(11) in Eq. (7), the PDEs along with the boundary conditions for the different perturbation powers of virtual parameter can be written as

$$
p_{1}^{0} p_{2}^{0}:\left\{\begin{array}{l}
\left(h_{0,0}\right)_{, t}-\omega / 2 k_{1}^{2}\left(h_{0,0}\right)_{x^{*} x^{*}}=0 \\
h_{0,0}\left(x^{*}=0, t\right)=D+A \cos [\omega t] \\
\left.\left(h_{0,0}\right)_{x^{*}}\right|_{x^{*} \rightarrow \infty}=0 .
\end{array}\right.
$$

zero or one. The solution can be expanded in series form using the two perturbation parameters from the homotopy equation as follows:

$$
h=\sum_{m=0}^{N_{p}} \sum_{n=0}^{N_{p}} p_{1}^{m} p_{2}^{n} h_{m, n} B_{\left[m+n \leq N_{p}\right]}
$$

where $N_{p}$ is the order of series expansion, $m$ and $n$ are incremental index variables and $B$ is a Boolean function. The Boolean function has value one when $\left[m+n \leq N_{p}\right]$, and zero otherwise. The second-order spatial derivative with zero coefficient (virtual term) in the LHS of Eq. (7) is the physical approximation of the first nonlinear term in the RHS. The coefficient zero can be replaced with parameter expansion to eliminate the secular terms generated from the first nonlinear term in the RHS of Eq. (7). The parameter expansion for coefficient zero can be written as

$$
p_{1}^{0} p_{2}^{1}:\left\{\begin{array}{l}
\stackrel{\left(h_{0,1}\right)_{, t}-\omega / 2 k_{1}^{2}\left(h_{0,1}\right)_{x^{*}} x^{*}}{=} \underbrace{\left[-w_{0,1}\left(h_{0,0}\right)_{x^{*} x^{*}}\right]}_{I_{0,1}^{1}}+\underbrace{\left[-s_{p} \sin [\omega t]\left(h_{0,0}\right)_{x^{*}}\right]}_{I_{0,1}^{2}} \\
h_{0,1}\left(x^{*}=0, t\right)=0 \\
\left.\left(h_{0,1}\right)_{x^{*}}\right|_{x^{*} \rightarrow \infty}=0 .
\end{array}\right.
$$

where $I_{1,0}\left(=I_{1,0}^{1}+I_{1,0}^{2}+I_{1,0}^{3}\right)$ and $I_{0,1}\left(=I_{0,1}^{1}+I_{0,1}^{2}\right)$ are the inhomogeneous terms of $p_{1}^{1} p_{2}^{0}$ and $p_{1}^{0} p_{2}^{1}$, respectively; $k_{1}$ is the wave number defined as

$$
k_{1}=\sqrt{\frac{\omega}{2 w_{0,0}}} .
$$

Solution for the zeroth-order equation $\left(p_{1}^{0} p_{2}^{0}\right)$ is given by

$$
h_{0,0}\left(x^{*}, t\right)=D+A e^{-k_{1} x^{*}} \cos \left[\omega t-k_{1} x^{*}\right] .
$$


Substituting the zeroth-order solution $h_{0,0}$ [Eq. (16)] in Eq. (13), the inhomogeneous term $I_{1,0}$ can be obtained as

$$
\begin{aligned}
& I_{1,0}=\frac{A^{2} k_{1}^{2} K}{\eta_{e}} e^{-2 k_{1} x^{*}}-\frac{8 A^{4} k_{1}^{4} K}{3 \eta_{e}} e^{-4 k_{1} x^{*}} \cos \left[4 \omega t-4 k_{1} x^{*}\right] \\
& -\frac{9 A^{3} k_{1}^{4} K D}{\eta_{e}} e^{-3 k_{1} x^{*}} \cos \left[3 \omega t-3 k_{1} x^{*}\right] \\
& -\frac{8 A^{4} k_{1}^{4} K}{3 \eta_{e}} e^{-4 k_{1} x^{*}} \cos \left[2 \omega t-2 k_{1} x^{*}\right] \\
& -\frac{8 A^{2} k_{1}^{4} K D^{2}}{\eta_{e}} e^{-2 k_{1} x^{*}} \cos \left[2 \omega t-2 k_{1} x^{*}\right] \\
& -\frac{3 A^{3} k_{1}^{4} K D}{\eta_{e}} e^{-3 k_{1} x^{*}} \cos \left[\omega t-k_{1} x^{*}\right] \\
& -\frac{2 A^{2} k_{1}^{2} K}{\eta_{e}} e^{-2 k_{1} x^{*}} \sin \left[2 \omega t-2 k_{1} x^{*}\right] \\
& -\frac{2 A^{4} k_{1}^{4} K}{\eta_{e}} e^{-4 k_{1} x^{*}} \sin \left[2 \omega t-2 k_{1} x^{*}\right] \\
& -\frac{4 A^{3} k_{1}^{4} K D}{\eta_{e}} e^{-3 k_{1} x^{*}} \sin \left[\omega t-k_{1} x^{*}\right] \\
& -\frac{A k_{1}\left(4 D^{3} k_{1}^{3} K+3 \eta_{e} \alpha_{0,0}\right)}{3 \eta_{e}} \underbrace{e^{-k_{1} x^{*}} \cos \left[\omega t-k_{1} x^{*}\right]}_{\text {Secular term }} \\
& +\frac{A k_{1}\left(-2 k_{1} K D+2 k_{1} \eta_{e} w_{1,0}+\eta_{e} \alpha_{0,0}\right)}{\eta_{e}} \\
& \underbrace{e^{-k_{1} x^{*}} \sin \left[\omega t-k_{1} x^{*}\right]}_{\text {Secular term }} \text {. }
\end{aligned}
$$

There are two secular terms $\left[e^{-k_{1} x^{*}} \cos \left(\omega t-k_{1} x^{*}\right)\right.$ and $\left.e^{-k_{1} x^{*}} \sin \left(\omega t-k_{1} x^{*}\right)\right]$ in $I_{1,0}$. Forcing the coefficients of the secular terms to zero for the requirement of no secular term in $I_{1,0}$, the following Eqs. (18) and (19) can be obtained:

$$
\begin{gathered}
4 D^{3} k_{1}^{3} K+3 \eta_{e} \alpha_{0,0}=0, \\
-2 k_{1} K D+2 k_{1} \eta_{e} w_{1,0}+\eta_{e} \alpha_{0,0}=0 .
\end{gathered}
$$

Solving Eqs. (18) and (19), $\alpha_{0,0}$ and $w_{1,0}$ can be written as follows:

$$
\begin{gathered}
\alpha_{0,0}=-\frac{4 D^{3} k_{1}^{3} K}{3 \eta_{e}}, \\
w_{1,0}=\frac{K D}{\eta_{e}}+\frac{2}{3} \frac{D^{3} k_{1}^{2} K}{\eta_{e}} .
\end{gathered}
$$

The solution $h_{1,0}\left(x^{*}, t\right)$ for Eq. (13) can be written as an addition of the particular integral $h_{1,0}^{P I}\left(x^{*}, t\right)$ and the complementary function $h_{1,0}^{C F}\left(x^{*}, t\right)$. Particular integral for the first-order equation [Eq. (13)] can be given as

$$
\begin{aligned}
& h_{1,0}^{P I}\left(x^{*}, t\right) \\
&=-\frac{A^{2} k_{1}^{2} K}{2 \eta_{e} \omega} e^{-2 k_{1} x^{*}}-\frac{A^{2} k_{1}^{2} K}{\eta_{e} \omega} e^{-2 k_{1} x^{*}} \cos \left[2 \omega t-2 k_{1} x^{*}\right] \\
&+ \frac{A^{4} k_{1}^{4} K}{18 \eta_{e} \omega} e^{-4 k_{1} x^{*}} \cos \left[2 \omega t-2 k_{1} x^{*}\right] \\
&+\frac{A^{3} D k_{1}^{4} K}{5 \eta_{e} \omega} e^{-3 k_{1} x^{*}} \cos \left[\omega t-k_{1} x^{*}\right] \\
&+\frac{2 A^{4} k_{1}^{4} K}{9 \eta_{e} \omega} e^{-4 k_{1} x^{*}} \sin \left[4 \omega t-4 k_{1} x^{*}\right] \\
&+\frac{3 A^{3} k_{1}^{4} K D}{2 \eta_{e} \omega} e^{-3 k_{1} x^{*}} \sin \left[3 \omega t-3 k_{1} x^{*}\right] \\
&+\frac{7 A^{4} k_{1}^{4} K}{18 \eta_{e} \omega} e^{-4 k_{1} x^{*}} \sin \left[2 \omega t-2 k_{1} x^{*}\right] \\
&+\frac{4 A^{2} k_{1}^{4} K D^{2}}{\eta_{e} \omega} e^{-2 k_{1} x^{*}} \sin \left[2 \omega t-2 k_{1} x^{*}\right] \\
&+\frac{11 A^{3} k_{1}^{4} K D}{10 \eta_{e} \omega} e^{-3 k_{1} x^{*}} \sin \left[\omega t-k_{1} x^{*}\right] .
\end{aligned}
$$

See the Supplementary material for the sample derivations of particular integrals, complementary functions and process of enforcing the boundary condition in the solution for PDEs. The solution $h_{1,0}\left(x^{*}, t\right)$ for the $p_{1}^{1} p_{2}^{0}$ equation [Eq. (13)] can be given as

$$
\begin{aligned}
& h_{1,0}\left(x^{*}, t\right) \\
&=\frac{A^{2} k_{1}^{2} K}{2 \eta_{e} \omega}-\frac{A^{2} k_{1}^{2} K}{2 \eta_{e} \omega} e^{-2 k_{1} x^{*}} \\
& \quad-\frac{A^{2} k_{1}^{2} K}{\eta_{e} \omega} e^{-2 k_{1} x^{*}} \cos \left[2 \omega t-2 k_{1} x^{*}\right] \\
&+\frac{A^{4} k_{1}^{4} K}{18 \eta_{e} \omega} e^{-4 k_{1} x^{*}} \cos \left[2 \omega t-2 k_{1} x^{*}\right] \\
&+\frac{A^{2} k_{1}^{2} K}{\eta_{e} \omega} e^{-\sqrt{2} k_{1} x^{*}} \cos \left[2 \omega t-\sqrt{2} k_{1} x^{*}\right] \\
&-\frac{A^{4} k_{1}^{4} K}{18 \eta_{e} \omega} e^{-\sqrt{2} k_{1} x^{*}} \cos \left[2 \omega t-\sqrt{2} k_{1} x^{*}\right] \\
&+\frac{A^{3} k_{1}^{4} K D}{5 \eta_{e} \omega} e^{-3 k_{1} x^{*}} \cos \left[\omega t-k_{1} x^{*}\right] \\
&-\frac{A^{3} k_{1}^{4} K D}{5 \eta_{e} \omega} e^{-k_{1} x^{*}} \cos \left[\omega t-k_{1} x^{*}\right] \\
&-\frac{2 A^{4} k_{1}^{4} K}{9 \eta_{e} \omega} e^{-2 k_{1} x^{*}} \sin \left[4 \omega t-2 k_{1} x^{*}\right] \\
&+\frac{2 A^{4} k_{1}^{4} K}{9 \eta_{e} \omega} e^{-4 k_{1} x^{*}} \sin \left[4 \omega t-4 k_{1} x^{*}\right]
\end{aligned}
$$




$$
\begin{aligned}
& +\frac{3 A^{3} k_{1}^{4} K D}{2 \eta_{e} \omega} e^{-3 k_{1} x^{*}} \sin \left[3 \omega t-3 k_{1} x^{*}\right] \\
& -\frac{3 A^{3} D k_{1}^{4} K}{2 \eta_{e} \omega} e^{-\sqrt{3} k_{1} x^{*}} \sin \left[3 \omega t-\sqrt{ } 3 k_{1} x^{*}\right] \\
& +\frac{7 A^{4} k_{1}^{4} K}{18 \eta_{e} \omega} e^{-4 k_{1} x^{*}} \sin \left[2 \omega t-2 k_{1} x^{*}\right] \\
& +\frac{4 A^{2} k_{1}^{4} K D^{2}}{\eta_{e} \omega} e^{-2 k_{1} x^{*}} \sin \left[2 \omega t-2 k_{1} x^{*}\right] \\
& -\frac{7 A^{4} k_{1}^{4} K}{18 \eta_{e} \omega} e^{-\sqrt{2} k_{1} x^{*}} \sin \left[2 \omega t-\sqrt{ } 2 k_{1} x^{*}\right] \\
& -\frac{4 A^{2} k_{1}^{4} K D^{2}}{\eta_{e} \omega} e^{-\sqrt{2} k_{1} x^{*}} \sin \left[2 \omega t-\sqrt{ } 2 k_{1} x^{*}\right] \\
& +\frac{11 A^{3} k_{1}^{4} K D}{10 \eta_{e} \omega} e^{-3 k_{1} x^{*}} \sin \left[\omega t-k_{1} x^{*}\right] \\
& -\frac{11 A^{3} k_{1}^{4} K D}{10 \eta_{e} \omega} e^{-k_{1} x^{*}} \sin \left[\omega t-k_{1} x^{*}\right] .
\end{aligned}
$$

Substituting the zeroth-order solution [Eq. (16)] in Eq. (14), the inhomogeneous term $I_{0,1}$ can be obtained as

$$
\begin{aligned}
\mathrm{I}_{0,1}= & -\frac{A k_{1} s_{p}}{2} e^{-k_{1} x^{*}} \cos \left[k_{1} x^{*}\right]+\frac{A k_{1} s_{p}}{2} e^{-k_{1} x^{*}} \\
& \cos \left[2 \omega t-k_{1} x^{*}\right] \\
& +\frac{A k_{1} s_{p}}{2} e^{-k_{1} x^{*}} \sin \left[k_{1} x^{*}\right]+\frac{A k_{1} s_{p}}{2} e^{-k_{1} x^{*}} \\
& \sin \left[2 \omega t-k_{1} x^{*}\right] \\
& +2 A k_{1}^{2} w_{0,1} \underbrace{e^{-k_{1} x^{*}} \sin \left[\omega t-k_{1} x^{*}\right]}_{\text {Secular term }}
\end{aligned}
$$

Forcing the coefficients of the secular terms $\left[e^{-k_{1} x^{*}} \sin \left(\omega t-k_{1} x^{*}\right)\right]$ to zero, $w_{0,1}$ can be given as

$$
w_{0,1}=0 \text {. }
$$

The solution $h_{0,1}\left(x^{*}, t\right)$ for the $p_{1}^{0} p_{2}^{1}$ equation [Eq. (13)] can be given as

$$
\begin{aligned}
& h_{0,1}\left(x^{*}, t\right) \\
&=\frac{A k_{1} s_{p}}{2 \omega}-\frac{A k_{1} s_{p}}{2 \omega} e^{-k_{1} x^{*}} \cos \left[k_{1} x^{*}\right] \\
&-\frac{A k_{1} s_{p}}{2 \omega} e^{-k_{1} x^{*}} \cos \left[2 \omega t-k_{1} x^{*}\right] \\
&+\frac{A k_{1} s_{p}}{2 \omega} e^{-\sqrt{2} k_{1} x^{*}} \cos \left[2 \omega t-\sqrt{2} k_{1} x^{*}\right] \\
&-\frac{A k_{1} s_{p}}{2 \omega} e^{-k_{1} x^{*}} \sin \left[k_{1} x^{*}\right] \\
&+\frac{A k_{1} s_{p}}{2 \omega} e^{-k_{1} x^{*}} \sin \left[2 \omega t-k_{1} x^{*}\right] \\
&-\frac{A k_{1} s_{p}}{2 \omega} e^{-\sqrt{2} k_{1} x^{*}} \sin \left[2 \omega t-\sqrt{2} k_{1} x^{*}\right] .
\end{aligned}
$$

Solving the linear PDEs for $h_{0,0}, h_{1,0}, h_{0,1}, \ldots$ the higherorder solution can be obtained using $p_{1} \rightarrow 1$ and $p_{2} \rightarrow 1$ in Eq. (9). The solution can be written as

$$
\begin{aligned}
h & =\lim _{p_{1} \rightarrow 1 p_{2} \rightarrow 1} \sum_{m=0}^{N_{p}} \sum_{n=0}^{N_{p}} p_{1}^{m} p_{2}^{n} h_{m, n} B_{\left[m+n \leq N_{p}\right]} \\
& =h_{0,0}+h_{1,0}+h_{0,1} .
\end{aligned}
$$

Similarly, the implicit equation for $w_{0,0}$ can be obtained using $p_{1} \rightarrow 1$ and $p_{2} \rightarrow 1$ in Eq. (10) as follows:

$$
\begin{aligned}
w_{0,0} & =\sum_{m=0}^{N_{p}} \sum_{n=0}^{N_{p}} p_{1}^{m} p_{2}^{n} w_{m, n} B_{\left[m+n \leq N_{p}\right]} B_{[m+n>0]} \\
& =w_{1,0}+w_{0,1}+\ldots
\end{aligned}
$$

Since the solution is up to only first order $(m+n \leq 1)$, the implicit equation for $w_{0,0}$ becomes

$$
w_{0,0}=w_{1,0}+w_{0,1}=\frac{K D}{\eta_{e}}+\frac{2}{3} \frac{D^{3} k_{1}^{2} K}{\eta_{e}} .
$$

\section{Discussion and solution comparisons}

The parameter $w_{0,0}$ becomes influential since the wave number $k_{1}$ is dependent on it. The parameter $w_{0,0}$ is dependent on the solution order since more terms are added to Eq. (29) with increasing order of the solution. The amplitude and phase of water table wave are dependent on the hydraulic conductivity of the aquifer $(K)$, mean sea level above aquifer bottom $(D)$, effective porosity of the aquifer $\left(\eta_{e}\right)$, amplitude $(A)$, angular frequency of the tidal wave $(\omega)$ and slope of the beach face $(\beta)$. The magnitude of $h_{0,1}$ term is dependent on slope of the beach face since all terms are multiplied by $A k_{1} s_{p} / \omega$. Similarly from the parameters $w_{0,0}$ and solution $h_{1,0}$, it is evident that $K D /$ $\eta_{e}, D^{3} k_{1}^{2} K / \eta_{e}, A^{2} k_{1}^{2} K /\left(\eta_{e} \omega\right), A^{4} k_{1}^{4} K /\left(\eta_{e} \omega\right)$ and $A^{3} k_{1}^{4} K D /\left(\eta_{e} \omega\right)$ are the controlling parameter groups of the groundwater waves.

The solution accuracy is validated by comparing the present solution with Kong et al [9] and Roberts et al [19] analytical solutions, and a 2D numerical solution (figure 2, tables 1 and 2). The analytical solution of Kong et al [9] is up to third order, and the Roberts et al [19] solution is up to sixth order. A two-dimensional numerical solution of Richards equations is obtained by 2D FEFLOW simulation for the vertical cross-section. FEFLOW is a well-established finite-element-based variably saturated flow and transport simulator. The unsaturated flow Richards model is simulated for beach slope of $30^{\circ}$. The tidal oscillations boundary condition is defined by a time series for the hydraulic head. The relationship between water content and the suction pressure is obtained using the Van Genuchten [20] model for water retention curve. The scale parameter value of $14.5(1 / \mathrm{m})$ and slope parameter value of 2.68 are used for the Van Genuchten model. The values of these parameters for sand are taken from probability-distributionbased tables of Carsel and Parrish [21]. The numerical 


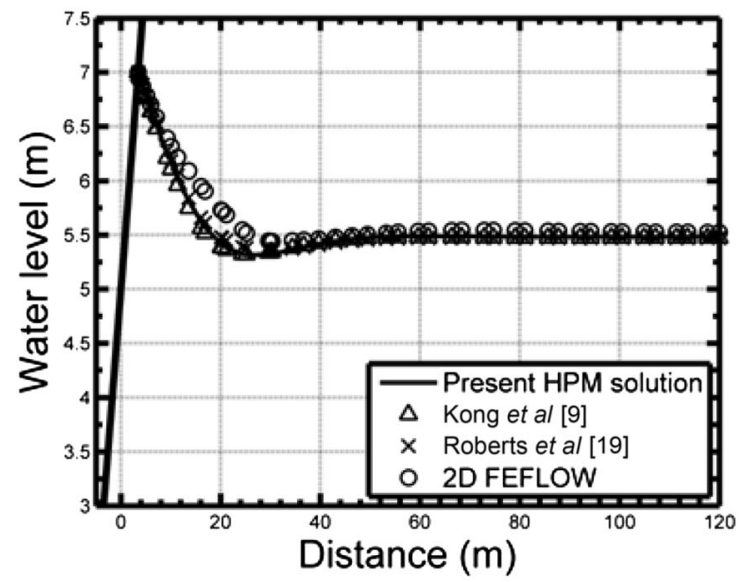

(a) $t=0 \mathrm{~h}$

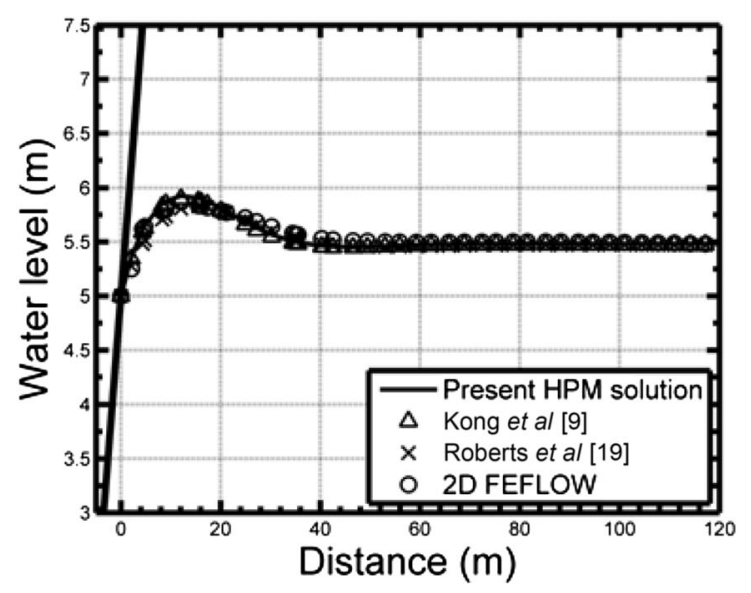

(c) $t=3 \mathrm{~h}$

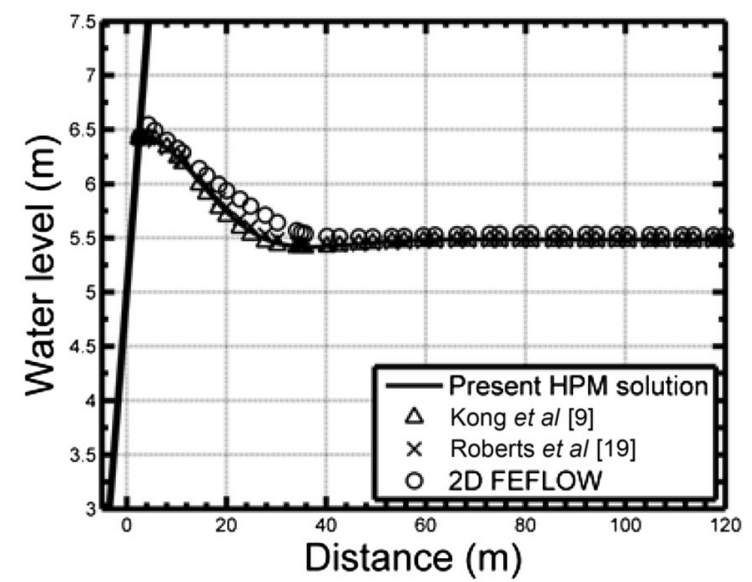

(b) $t=1.5 \mathrm{~h}$

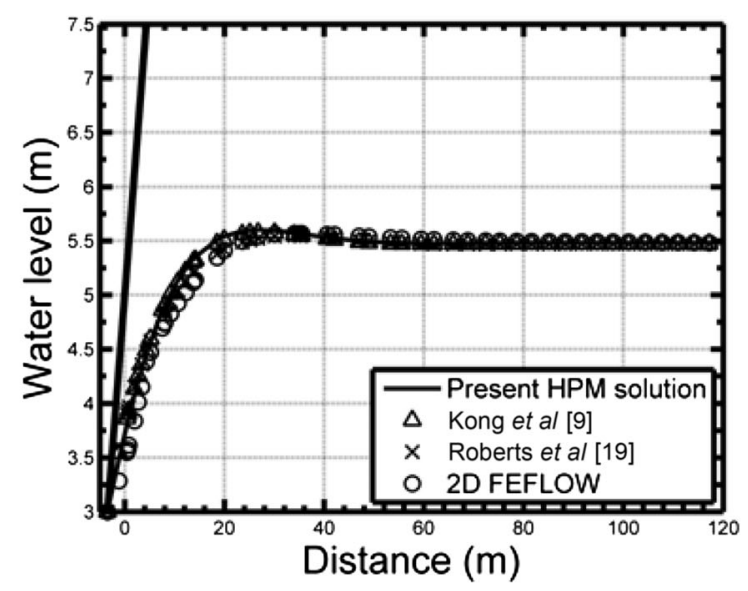

(d) $t=6 \mathrm{~h}$

Figure 2. Comparison of the homotopy perturbation solution for the higher-order Boussinesq equation with Kong et al [9], Roberts et al [19] and 2D FEFLOW solutions at different time instances for a tidal wave with time period $=12 \mathrm{~h}, D=5 \mathrm{~m}, A=2 \mathrm{~m}, \eta_{e}=0.3$, $K=0.0005 \mathrm{~m} / \mathrm{s}$ and $\beta=30^{\circ}$.

errors are removed with a grid convergence test by increasing the number of elements. The parameters provided in Kong et al [9] are used for solution comparison: $\eta_{e}=0.3, K=5 \times 10^{-4} \mathrm{~m} / \mathrm{s}, D=5 \mathrm{~m}, A=2 \mathrm{~m}, \quad$ and $\omega=2 \pi /(12 * 60 * 60) \mathrm{rad} / \mathrm{s}$.

The value of implicit parameter $w_{0,0}$ is $0.0094 \mathrm{~m}^{2} / \mathrm{s}$ [obtained by solving Eq. (29)] for the specified parameter values. The solution plots are provided for times $0 \mathrm{~h}, 1.5 \mathrm{~h}, 3 \mathrm{~h}$, and $6 \mathrm{~h}$. Roberts et al [19] solution methodology is based on the inclusion and exclusion of terms based on relative sizes of the magnitudes. Kong et al [9] solution is based on non-dimensional perturbation parameters with secular term removal performed using a perturbation expansion for boundary condition. The perturbation expansion of boundary condition by Kong et al [9] introduces a small correction to the boundary The handling of secular terms in the present solution is unique and does not introduce any correction at the boundary.
Table 1. Mean absolute percentage error (MAPE) between the present solution and Kong et al [9], Roberts et al [19] and 2D FEFLOW numerical solution.

\begin{tabular}{lccc}
\hline Time & $\begin{array}{c}\text { Kong et al } \\
\text { [9] (up to } \\
\text { third order) }\end{array}$ & $\begin{array}{c}\text { Roberts } \text { et al } \\
\text { [19] (up to } \\
\text { sixth order) }\end{array}$ & $\begin{array}{c}\text { Present } \\
\text { solution (up to } \\
\text { first order) }\end{array}$ \\
\hline$t=0 \mathrm{~h}$ & 0.1651 & 0.2307 & 0.8582 \\
$t=1.5 \mathrm{~h}$ & 0.1404 & 0.2088 & 0.8053 \\
$t=3 \mathrm{~h}$ & 0.1499 & 0.2733 & 2.1766 \\
$t=6 \mathrm{~h}$ & 0.1967 & 0.3683 & 2.3425 \\
\hline
\end{tabular}

Parameters: time period $=12 \mathrm{~h}, D=5 \mathrm{~m}, A=2 \mathrm{~m}, \eta_{e}=0.3, K=$ $0.0005 \mathrm{~m} / \mathrm{s}$ and $\beta=30^{\circ}$.

Moreover, the homotopy-based first-order solution of second-order Boussinesq equation is in good agreement with the solutions by Kong et al [9] and Roberts et al [19]. From the present solution, it is evident that with the minimum 
Table 2. Mean absolute percentage error (MAPE) between the numerical solution and Kong et al [9], Roberts et al [19] and the present solution.

\begin{tabular}{|c|c|c|c|c|}
\hline Solution & $t=0 \mathrm{~h}(\%)$ & $t=1.5 \mathrm{~h}(\%)$ & $t=3 \mathrm{~h}(\%)$ & $t=6 \mathrm{~h}(\%)$ \\
\hline Kong et al [9] (up to third order) & 0.8778 & 0.8091 & 2.0833 & 2.2650 \\
\hline Roberts et al [19] (up to sixth order) & 0.8006 & 0.9726 & 2.9411 & 6.0992 \\
\hline Present solution (up to first order) & 0.8582 & 0.8053 & 2.1766 & 2.3062 \\
\hline
\end{tabular}

Parameters: time period $=12 \mathrm{~h}, D=5 \mathrm{~m}, A=2 \mathrm{~m}, \eta_{e}=0.3, K=0.0005 \mathrm{~m} / \mathrm{s}$ and $\beta=30^{\circ}$.

order a more accurate solution can be derived from higherorder Boussinesq equation compared with previous analytical solutions based on the ordinary Boussinesq equation (table 2). The second-order Boussinesq equation captures the streamline curvatures [15]. More accurate higher-order solutions for the second-order Boussinesq equation can be derived by extending the homotopy method with an additional auxiliary term for handling the additional secular term $\left[e^{-k_{1} x^{*}} \cos \left(\omega t+k_{1} x^{*}\right)\right]$ generated from the slope term. The homotopy solution is derived without any predefined physical parameters. However, the beach slope emerged as an implicit parameter from the solution process [Eq. (26)]. The parameter $s_{p}[=A \omega \cot (\beta)]$ is inversely proportional to beach slope angle. The value of $s_{p}$ becomes large for small beach slopes.

\subsection{Solution accuracy with increasing perturbation order}

The accuracy of the present solution with increasing order of the solution is analysed using the 2D FEFLOW solution. The mean absolute deviation $(M A D)$ of the solution orders is calculated using the following formulation:

$$
M A D_{m, n}=\frac{1}{n_{d}} \sum_{s=1}^{n_{d}}\left|h_{\text {num }}-\sum_{i=0 j=0}^{m, n} h_{i, j}\right|
$$

where $M A D_{m, n}$ is $M A D$ of the analytical $\sum_{i=0 j=0}^{m, n} h_{i, j}$ solution up to the order $p_{1}^{m} p_{2}^{n}$ with reference to the 2D FEFLOW numerical solution $h_{\text {num }}$ and $n_{d}$ is the number of data points, where $i, j$, and $s$ are incremental indices. The numerical solution for slope of $30^{\circ}$ is analysed. For time instance $t=0 \mathrm{~h}$, the sum of the absolute head deviations as obtained from the proposed solution and from the parallel FEFLOW codes is found to decrease with the increase in order of perturbation solution for the tested conditions, where $M A D_{0,0}, M A D_{1,0}$, and $M A D_{0,1}$, are calculated as $0.4701 \mathrm{~m}, 0.2956 \mathrm{~m}$, and $0.0480 \mathrm{~m}$, respectively. The trend is similar for $t=1.5 \mathrm{~h}$ with $M A D_{0,0}, M A D_{1,0}$, and $M A D_{0,1}$, calculated as $0.4702 \mathrm{~m}, 0.2972 \mathrm{~m}$, and $0.0449 \mathrm{~m}$, respectively. The $M A D$ values for $t=3 \mathrm{~h}$ are obtained as $0.3640 \mathrm{~m}, 0.1922 \mathrm{~m}$, and $0.1158 \mathrm{~m}$, correspondingly. The $M A D$ values for $t=6 \mathrm{~h}$ are obtained as $0.3503 \mathrm{~m}, 0.1911 \mathrm{~m}$, and $0.1183 \mathrm{~m}$, correspondingly. From the $M A D$ values, it is evident that the sum of absolute head deviations of homotopy perturbation solution and FEFLOW solutions decreases with increasing order of homotopy perturbation solution.

\section{Conclusions}

An analytical solution is provided for water table variations driven by tidal oscillations in coastal aquifers. The homotopy-perturbation-based solution for higher-order Boussinesq equation is applicable for coastal aquifers with a sloping beach face. A unique secular term removal process is applied using parameter expansion and an auxiliary term. The solution does not require any predefined perturbation parameters. However, some implicit physical parameters emerge from the solution process. The implicit parameter $w_{0,0}$ influences the amplitude and phase of the water table wave oscillations. The beach slope $\left[s_{p}=A \omega \cot (\beta)\right]$ influences the magnitude of the solution. The parameter $s_{p}$ becomes large for mild beach slopes. Hence, the solution accuracy is good with increasing beach slopes. The solution is provided up to first order $(m+n \leq 1)$ and can be easily extended to higher order with an additional auxiliary term for handling the secular term. This approach is limited to relatively steeper slopes. Tidal dynamics in flat beach face requires the solution of full-scale governing equation.

\section{List of symbols}

$h \quad$ local water table height $(\mathrm{m})$

$h_{m, n} \quad$ water table height of order $m, n$ where $m$ and $n$ are incremental index variables $(\mathrm{m})$

$h_{, x} \quad$ partial differential of $h$ with respect to $x(-)$

$h{ }_{t} \quad$ partial differential of water table height $h$ with respect to $t\left(\mathrm{~m} \mathrm{~s}^{-1}\right)$

(), ${ }_{x}$ partial differential of the expression inside the braces with respect to $x(-)$

(), $x x$ second-order partial differential of the expression inside the braces with respect to $x(-)$

(), $x x x$ third-order partial differential of the expression inside the braces with respect to $x$

$k_{1} \quad \sqrt{\omega / 2 w_{0,0}}\left(\mathrm{~m}^{-1}\right)$

$x \quad$ longitudinal coordinate $(\mathrm{m})$

$t \quad$ time (s)

$p_{1}, p_{2} \quad$ embedding virtual parameters (-) 


\begin{tabular}{|c|c|}
\hline$w_{m, n}$ & $\begin{array}{l}\text { parameters used in the parameter expansion }\left(\mathrm{m}^{2}\right. \\
\left.\mathrm{s}^{-1}\right)\end{array}$ \\
\hline$A$ & amplitude of the tidal oscillations (m) \\
\hline$D$ & mean sea level above aquifer bottom (m) \\
\hline$I_{m, n}$ & inhomogeneous terms $\left(\mathrm{m} \mathrm{s}^{-1}\right)$ \\
\hline$K$ & hydraulic conductivity of the aquifer $\left(\mathrm{m} \mathrm{s}^{-1}\right)$ \\
\hline$s_{p}$ & $A \omega \cot [\beta]\left(\mathrm{m} \mathrm{s}^{-1}\right)$ \\
\hline$x^{*}$ & $\begin{array}{l}x-x_{0}(t) \text {, transformed coordinate for moving } \\
\text { boundary, where } x_{0}(t) \text { is } \cot (\beta) A \cos (\omega t)(\mathrm{m})\end{array}$ \\
\hline & coefficient of auxiliary term $\left(\mathrm{m} \mathrm{s}^{-1}\right)$ \\
\hline$\alpha_{m, n}$ & $\begin{array}{l}\text { parameters used in the parameter expansion of } \alpha \\
\left(\mathrm{m} \mathrm{s}^{-1}\right)\end{array}$ \\
\hline$\eta_{e}$ & effective porosity of the aquifer $(-)$ \\
\hline & angular frequency of the tidal oscillations (s \\
\hline
\end{tabular}

\section{Acknowledgements}

We are grateful to Dr Melanie E Roberts, Research Scientist, IBM, Melbourne, Australia, for providing us the sixth-order solution for validation. Funding was provided by Science and Engineering Research Board (Grant No. SB/FTP/ETA-0356/2013).

\section{References}

[1] Jha M K and Singh A 2014 Application of genetic algorithm technique to inverse modeling of tide-aquifer interaction. Environ. Earth Sci. 71(8): 3655-3672

[2] Nielsen P 1990 Tidal dynamics of the water table in beaches. Water Resour. Res. 26(9): 2127-2134

[3] Parlange J Y, Stagnitti F, Starr J L and Braddock R D 1984 Free-surface flow in porous media and periodic solution of the shallow-flow approximation. J. Hydrol. 70(1-4): 251-263

[4] Dagan G 1967 Second-order theory of shallow free-surface flow in porous media. Q. J. Mech. Appl. Math. 20(4): 517-526

[5] Li L, Barry D A, Stagnitti F, Parlange J and Jeng D 2000 Beach water table fluctuations due to spring-neap tides: moving boundary effects. Adv. Water Res. 23(8): 817-824

[6] Teo H T, Jeng D S, Seymour B R, Barry D A and Li L 2003 A new analytical solution for water table fluctuations in coastal aquifers with sloping beaches. Adv. Water Res. 26(12): 1239-1247

[7] Jeng D S, Barry D A, Seymour B R, Dong P and Li L 2005 Two-dimensional approximation for tide-induced watertable fluctuations in a sloping sandy beach. Water Resour. Res. 28(10): 1040-1047

[8] Song Z, Li L, Kong J and Zhang H 2007 A new analytical solution of tidal water table fluctuations in a coastal unconfined aquifer. J. Hydrol. 340(3-4): 256-260

[9] Kong J, Song Z, Xin P and Shen C 2011 A new analytical solution for tide-induced groundwater fluctuations in an unconfined aquifer with a sloping beach. China Ocean Eng. 25(3): 479-494

[10] Cartwright N, Nielsen P, Li L and Callaghan D 2003 Watertable waves in unconfined aquifers: sloping boundary effects [online]. In: Kench P S and Hume T M (Eds.) Coasts \& Ports 2003 Australasian Conference: Proceedings of the 16th Australasian Coastal and Ocean Engineering Conference, the 9th Australasian Port and Harbour Conference and the Annual New Zealand Coastal Society Conference. Barton, A.C.T.: Institution of Engineers, Australia, pp. 918-926

[11] Stojsavljevic J D, Jeng D S, Seymour B R and Pokrajac D 2012 Higher order analytical solutions of water table fluctuations in coastal aquifers. Groundwater 50(2): 301-307, doi:10.1111/j.1745-6584.2011.00820.x

[12] Fenton J 1990 Calculating seepage flow with a free surface: some old methods and some new ones. Proc. N.Z. Geomech. Soc. 16: 31-36

[13] Nielsen P, Aseervatham R, Fenton J D and Perrochet P 1997 Groundwater waves in aquifers of intermediate depths. Adv. Water Res. 20(1): 37-43

[14] Liu P L F and Wen J 1997 Nonlinear diffusive surface waves in porous media. J. Fluid Mech. 347(1): 119-139

[15] Castro-Orgaz O and Dey S 2014 Second-order shallow-flow theory and Dupuit approximation for phreatic aquifers. $J$. Hydraul. Eng-ASCE 140(9): 04014040

[16] Song H and Longbin T 2007 Homotopy analysis of 1D unsteady, nonlinear groundwater flow through porous media. J. Coastal Res. 50: 292-295

[17] He J H 1999 Homotopy perturbation technique. Comput. Methods Appl. Mech. 178(3-4): 257-262

[18] He J H 2012 Homotopy perturbation method with an auxiliary term. Abstr. Appl. Anal. 2012(2012): 1-7

[19] Roberts M E, Trefry M G, Fowkes N, Bassom A P and Abbott P C 2011 Water-table response to tidal forcing at sloping beaches. J. Eng. Math. 69(4): 291-311, doi: 10.1007/ s10665-010-9407-7

[20] Van Genuchten M T 1980 A closed-form equation for predicting the hydraulic conductivity of unsaturated soils. Soil. Sci. Soc. Am. J. 44(5): 892-898

[21] Carsel R F and Parrish R S 1988 Developing joint probability distributions of soil water retention characteristics. Water Resour. Res. 24(5): 755-769, doi: 10.1029/WR024i005p00755 